\title{
Nanocomposite of Poly(Lactic Acid)/Cellulose Nanocrystals: Effect of CNC Content on the Polymer Crystallization Kinetics
}

\author{
Mauro Vestena, ${ }^{*, a b}$ Idejan P. Gross, ${ }^{a}$ Carmen M. O. Müller ${ }^{c}$ and Alfredo T. N. Pires ${ }^{a}$ \\ ${ }^{a}$ Grupo de Estudo em Materias Poliméricos (POLIMAT), Departamento de Química, Universidade \\ Federal de Santa Catarina (UFSC), 88040-900 Florianópolis-SC, Brazil \\ ${ }^{b}$ Departamento Acadêmico de Química, Universidade Tecnológica Federal do Paraná(UTFPR), \\ Campus Pato Branco, 85503-390 Pato Branco-PR, Brazil \\ ${ }^{c}$ Departamento de Ciência e Tecnologia de Alimentos, Universidade Federal de Santa Catarina \\ (UFSC), 88034-001 Florianópolis-SC, Brazil
}

\begin{abstract}
The barrier and mechanical properties of polymer matrices are affected by the polymer crystallization kinetics and the incorporation of nanoparticles can modify the nucleation density as well as the rate and mechanism of crystallization. In this study, a nanocomposite of poly(lactic acid) (PLA) containing cellulose nanocrystals (CNC) was prepared in order to evaluate the effect of the CNC content on the crystallization kinetics of the polymer matrix. Dimethylformamide, a solvent of PLA, was used to replace the aqueous medium for the dispersion of the CNC, to facilitate the preparation of PLA films with dispersed CNC. Higher levels of CNC in the films induced an increase in the crystallization rate and reduced the degree of crystallinity without affecting the dimensions of the crystal lattice. In conclusion, the incorporation of CNC into PLA influences the crystallization kinetics, which significantly affects the PLA processing conditions.
\end{abstract}

Keywords: cellulose nanocrystals, poly(lactic acid), crystallization kinetics, nanocomposites

\section{Introduction}

Particles with nanometric dimensions, such as carbon nanotubes, nanoclays and cellulose nanocrystals (CNC), have been used as reinforcing materials in polymer matrices. The addition of such particles alters the physicochemical characteristics of the matrix, which are related to specific properties associated with these materials. ${ }^{1}$ The polymer crystallization kinetics, for example, can be enhanced by adding nanoparticles, which also influences the crystalline morphology and rheological behavior. ${ }^{2}$

CNC are crystalline domains isolated from lignocellulosic fibers that are composed of cellulosic microfibrils surrounded by lignin and hemicellulose. A bleaching process is therefore required to remove the non-cellulosic components of the lignocellulosic fibers, followed by acid hydrolysis to isolate the CNC. These nanomaterials are of particular importance, considering that cellulose is one of the most abundant renewable resources and a biodegradable polymer. ${ }^{1,3,4}$

*e-mail: mauro@utfpr.edu.br
In this context, environmental concerns have led to an increase in studies using biopolymer matrices, such as poly(lactic acid) (PLA), a linear aliphatic biodegradable polymer, derived from renewable resources. It can be produced by polycondensation from lactic acid or the ring-opening polymerization of lactides (cyclic dimer of lactic acid), resulting in low or high molar weight, respectively. The presence of a stereocenter in the PLA repeating unit results in the optically active enantiomers $\operatorname{poly}(L$-lactic acid) (PLLA) and poly( $D$-lactic acid) (PDLA), or optically inactive poly $(D, L$-lactic acid) (PDLLA), as well as a racemic mixture. ${ }^{1,5}$

PLLA can be crystallized in an $\alpha$ (orthorhombic), $\beta$ (orthorhombic) or $\gamma$ (pseudo-orthorhombic) system, and the $\alpha$-structure is more stable. ${ }^{6}$ Commercial-grade PLA is made up of $L$-lactide and $D, L$-lactide copolymers. ${ }^{5,7,8}$

The mechanical properties of PLA are comparable with other commodity thermoplastics, like polystyrene (PS) and poly(ethylene terephthalate) (PET). Besides presenting good processability, with many potential applications, such as packaging, drug delivery, tissue engineering and biological scaffolds, PLA is a potential replacement for 
non-biodegradable polymers. ${ }^{8.9}$ The barrier and mechanical properties can be affected by polymer crystallization kinetics, through the incorporation of nanoparticles that modify the nucleation density, and the rate and mechanism associated with the crystallization. ${ }^{10,11}$

Mathew and Dufresne ${ }^{12}$ studied the crystallinity of plasticized starch films with sorbitol and reported an increase in the crystallinity of the films when they incorporated CNC. They attributed this behavior to an anchoring effect of the $\mathrm{CNC}$, which act as nucleating agents for starch and favor the crystallization process. Anglés and Dufresne ${ }^{13}$ reported that composite films of starch/CNC presented the phenomenon of transcrystallization, which consists of the preferential crystallization of amorphous polymeric chains during cooling at the surface of the CNC. Samir et al. ${ }^{14}$ studied the crystallization of poly(oxyethylene) films with the incorporation of $\mathrm{CNC}$ and observed that increasing the $\mathrm{CNC}$ content resulted in a decrease in the average diameter of the spherulites, due to a change in the nucleation rate. In a previous study, Samir et al. ${ }^{15}$ observed that the presence of CNC within the polymeric matrix does not affect the spherulitic growth rate of poly(oxyethylene) during its crystallization. In agreement with other studies, Yu et al. ${ }^{16}$ evaluated poly(3-hydroxybutyrate-co-3-hydroxyvalerate (PHBV)/CNC nanocomposites and observed a decrease in the degree of crystallinity with an increase in the $\mathrm{CNC}$ content, due to strong intermolecular hydrogen bonding interactions associated with the dispersion of the CNC in the PHBV matrix. Similar studies on the PLA matrix have demonstrated the influence of $\mathrm{CNC}$ on the PLA crystallization, leading to an improvement in the nanocomposite mechanical properties, ${ }^{17}$ with a reduction in the water and oxygen permeability. ${ }^{18-20}$ The mechanical reinforcement of the nanocomposite is a consequence of different effects, such as the CNC distribution in the matrix, intrinsic CNC characteristics (shape, aspect ratio) and the crystallization process. The reduction in the gas and vapor water permeability may be associated with decreased diffusion due to the presence of the $\mathrm{CNC}$ which increases of the tortuous path of the diffusing components. ${ }^{4}$

One approach to evaluating the crystallization process is to consider the isothermal crystallization kinetics, which can be described by the Avrami model. The Avrami model describes the uncrystallized polymer mass fraction as a function of time and determines the crystallization rate and the Avrami parameter, which correlates with the growth dimensions and the crystallization mechanism. Kamal and Khoshkava $^{21}$ studied PLA/CNC nanocomposites, applying a previous process, such as spray drying, freeze drying or spray freeze drying, to obtain the CNC suspensions. These processes modify the size and porosity of CNC agglomerates, and consequently affect the dispersion in the polymer matrix. Higher values for the crystallization rate, nucleation density and lower spherulite dimension were observed with porous and smaller agglomerates of $\mathrm{CNC}$. In this context, the evaluation of the nanocomposite crystallization process is a field of study of considerable interest, since it is dependent on the polymer matrix and the nanoparticles incorporated.

Considering that the $\mathrm{CNC}$ were isolated in an aqueous medium, their incorporation into a hydrophobic polymer, such as PLA, is not favorable. Several strategies have been used to try and achieve the dispersion of $\mathrm{CNC}$ in hydrophobic matrices, e.g., adding surfactants, CNC surface modification, polymer grafting and solvent exchange dispersion. The best dispersion of $\mathrm{CNC}$ in a polyhydroxybutyrate (PHB) matrix has been observed for solvent exchange dispersion (water to acetone and acetone to chloroform) using centrifugation processes., ${ }^{916,17,22-26}$

In this context, the aim of this study was to evaluate the effect of the presence of CNC isolated from sugarcane bagasse after solvent exchange (water to dimethylformamide) on the isothermal crystallization kinetics of PLA.

\section{Experimental}

\section{Materials}

Sugarcane bagasse, provided by regional ethanol producers (São Paulo, Brazil), was washed, dried and milled to yield particles that passed through a 30 mesh sieve. Poly(lactic acid) was purchased from NatureWorks LLC, Cargill under the brand name Ingeo 3251D. Sulfuric acid and sodium hydroxide were purchased from Lafan Química Fina and dimethylformamide (DMF) was supplied by Nuclear. All chemical reagents were used without further purification.

\section{Cellulose nanocrystals (CNC)}

Initially, the particulates of sugarcane bagasse (smaller than 30 mesh) were maintained in a $5 \%$ aqueous solution of $\mathrm{NaOH}\left(1: 20 \mathrm{~m} / \mathrm{v}\right.$ fiber/solution) at $85^{\circ} \mathrm{C}$ for $90 \mathrm{~min}$, under mechanical stirring. In the next step, the dispersion was filtered and the fibers washed until neutral $\mathrm{pH}$ and dried. The fibers were then ground and maintained in an aqueous solution of $5 \% \mathrm{NaOH}$ and $11 \% \mathrm{H}_{2} \mathrm{O}_{2}(1: 20 \mathrm{~m} / \mathrm{v})$, at $55^{\circ} \mathrm{C}$ for $90 \mathrm{~min}$, and the dispersion was filtered and the fibers washed until neutral $\mathrm{pH}$ and dried. Acid hydrolysis was carried out at $55^{\circ} \mathrm{C}$ for 30 min with $60 \mathrm{wt} . \% \mathrm{H}_{2} \mathrm{SO}_{4}$ under mechanical stirring. The suspension was diluted with water 
(five times the volume of the reaction mixture) at room temperature to stop the reaction, and after centrifugation the supernatant was removed. Distilled water was then added to the sediment, which was suspended under mechanical stirring, and the suspension was centrifuged until the appearance of the cloud point. This suspension of CNC was collected and treated using dialysis bags at neutral $\mathrm{pH}$. The CNC dispersion medium was changed from water to DMF, in order to solubilize the PLA. DMF was added to the aqueous suspension of $\mathrm{CNC}$ and the water eliminated through vacuum distillation, since the boiling point of DMF is greater than the boiling point of water.

Transmission electron microscopy (TEM)

Transmission electron microscopy analysis of the CNC was carried out using a JEOL-1011 TEM electron microscope operating at $80 \mathrm{kV}$. One drop $\left(0.002 \mathrm{~g} \mathrm{~mL}^{-1}\right)$ of the suspension of $\mathrm{CNC}$ was diluted in $2 \mathrm{~mL}$ of isopropyl alcohol and deposited on a grid coated with FORMVAR ${ }^{\circledR}$ film and after being almost dried it was stained by adding one drop (ca. $4 \mu \mathrm{L}$ ) of $3 \%$ uranyl acetate $(\mathrm{m} / \mathrm{v}$ ). The excess liquid was removed and this was followed by drying at room temperature. To evaluate the length and width, five images and one hundred nanoparticles were considered and the ImageJ software was used (values reported are average).

\section{Films of PLA/CNC}

PLA was dissolved in the DMF suspension of CNC, at compositions of $99 / 1,97 / 3$ and $95 / 5(\mathrm{~m} / \mathrm{m})$. In this procedure the PLA was added to the suspension of CNC in DMF and the heating system maintained the temperature above $70^{\circ} \mathrm{C}$, for the polymer solubilization. The dispersion of PLA and CNC in DMF was transferred to Petri dishes and maintained at $120{ }^{\circ} \mathrm{C}$ for solvent evaporation to obtain thin films.

Isothermal crystallization kinetics and X-ray diffraction

The use of differential scanning calorimetry (DSC) has been found to be suitable for investigating the crystallization kinetics and in this study a Shimadzu DSC-50 instrument was used. All experiments were performed under nitrogen gas flow $\left(50 \mathrm{~mL} \mathrm{~min}{ }^{-1}\right)$. The samples were heated from room temperature up to $200{ }^{\circ} \mathrm{C}$, at a rate of $20^{\circ} \mathrm{C} \mathrm{min}^{-1}$, and maintained at this temperature for $5 \mathrm{~min}$ to allow complete melting of the crystalline domains. The temperature was then decreased and the samples were kept at $130{ }^{\circ} \mathrm{C}$ to evaluate the exothermic heat involved in the crystallization process. Based on the crystallization enthalpy, the degree of crystallization was obtained (equation 1) from the DSC curve, where $X_{c}^{\infty}$ is the degree of crystallization of the samples, $\Delta H_{c}$ is the crystallization enthalpy of the PLA in the sample, which is equal to the melting enthalpy of PLA $\left(\Delta H_{m}\right), \Delta H_{m}{ }^{0}$ is the melting enthalpy of $100 \%$ crystalline PLA and is equal to $93 \mathrm{~J} \mathrm{~g}^{-1}, 7$ and $f_{P L A}$ is the weight fraction of PLA in the nanocomposite.

$X_{c}^{\infty}=\frac{\Delta H_{m}}{\Delta H_{m}^{0} f_{P L A}}$

From the crystallization enthalpy as a function of time determined from the DSC isotherm curve, based on the heat evolved in the crystallization process at different time intervals, the degree of crystallization, denoted by $X_{c}^{t}$, can be evaluated and the isothermal crystallization kinetics data were fitted according to the Avrami equation (equation 2), where $X_{t}$ denotes the crystallized fraction of PLA as a function of time $t$, and $K$ and $n$ are the Avrami kinetic parameters:

$X_{t}=\frac{X_{c}^{t}}{X_{c}^{\infty}}=1-e^{-(K t)^{n}}$

Polarized optical microscopy was used to investigate the size, morphology and characteristics of spherulite crystals during the crystallization process. The nanocomposites were cast between two glass slides and the physical characteristics of each sample were observed under crossed polarization using a Motic SMZ-168-BL microscope, with a data acquisition system (TCS pro500) and the software VMS 3.6.

$\mathrm{X}$-Ray patterns of the specimens were obtained on a Philips X'Pert diffractometer, with $\mathrm{Cu} \mathrm{K}_{\alpha}$ radiation $(\lambda=1.5418 \AA)$, operating at room temperature, $30 \mathrm{~mA}$ and $40 \mathrm{kV}$. The scanned region of 2 to $60^{\circ}(2 \theta)$ and a pitch of $0.05^{\circ} \mathrm{s}^{-1}$ were applied to evaluate the influence of the CNC in the PLA unit cell. Samples of neat PLA and PLA/CNC with different percentages of the components in the composition were melted at $200{ }^{\circ} \mathrm{C}$ and maintained at $130{ }^{\circ} \mathrm{C}$ for $60 \mathrm{~min}$.

\section{Results and Discussion}

\section{Suspensions of CNC in water and in DMF}

$\mathrm{CNC}$ in aqueous suspension have limited dispersion in water-insoluble polymer matrices such as PLA. Thus, changing the solvent medium is one way to prevent the agglomeration of CNC in non-aqueous solution and obtain nanocomposites of a hydrophobic polymer matrix. 
Solvent exchange is an interesting process since it avoids the drying step and consequent agglomeration of the CNC. Other processes have been described in the literature to maintain the stability of the suspension, such as CNC surface modification or the addition of surfactants, which increases the complexity and cost of the process. ${ }^{22-27}$

The process used to change solvent through vacuum distillation, evaluated in this study, was found to be an efficient approach to changing the $\mathrm{CNC}$ suspension medium and preventing the agglomeration of $\mathrm{CNC}$ in the non-aqueous suspension. It was possible to obtain stable suspensions of CNC in DMF with $0.013 \mathrm{~g} \mathrm{~mL}^{-1}$ and in aqueous medium with $0.018 \mathrm{~g} \mathrm{~mL}^{-1}$, as determined by the gravimetric method. The morphology and dimensions of the $\mathrm{CNC}$ were evaluated through transmission electron microscopy. Figure 1 shows the TEM micrographs of the $\mathrm{CNC}$ dispersed in water and in DMF, where similar dispersions without $\mathrm{CNC}$ agglomeration can be observed, suggesting that nanocomposites with a hydrophobic polymer matrix could be obtained. The $\mathrm{CNC}$, with a rod-like aspect, had an average length of $230 \pm 53 \mathrm{~nm}(\mathrm{~L})$,
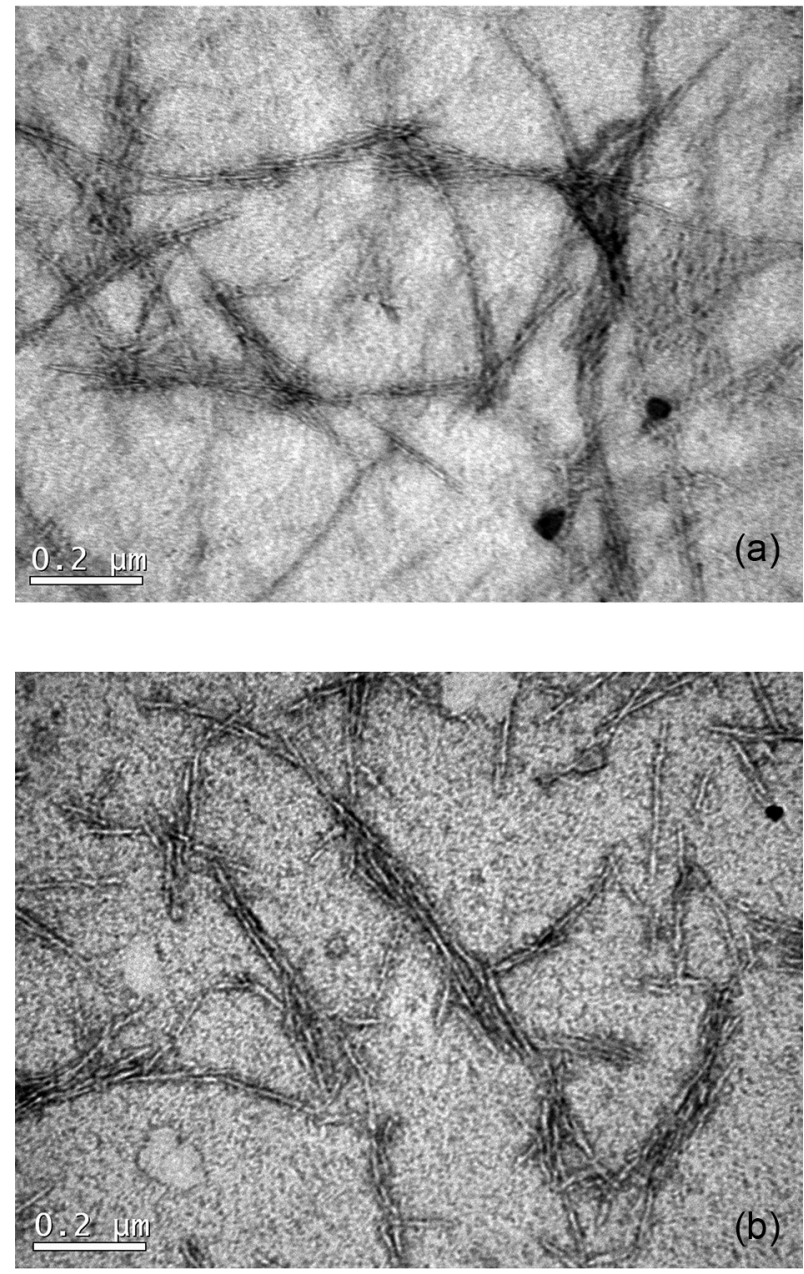

Figure 1. TEM micrographs of CNC dispersed in (a) water and (b) DMF. average diameter of $10 \pm 4 \mathrm{~nm}$, and aspect ratio (L / D) of 23. These values are in agreement with others reported in the literature. For CNC isolated from bagasse sugarcane, Teixeira et al. ${ }^{28}$ obtained average values for the length and diameter of $255 \pm 55$ and $8 \pm 3 \mathrm{~nm}$, respectively, while Mandal and Chakrabarty ${ }^{29}$ reported a shorter length $(170 \mathrm{~nm})$ and larger diameter $(35 \mathrm{~nm})$ compared with the values obtained in this study.

\section{Neat PLA and PLA/CNC nanocomposites}

The DSC curves (Figure 2a) obtained at constant temperature $\left(130^{\circ} \mathrm{C}\right)$ show the PLA crystallization peaks for neat PLA and PLA/CNC nanocomposites. In Figure 2a it can also be observed that the time required to reach the maximum crystallization rate decreases as the $\mathrm{CNC}$ content increases, which suggests that the $\mathrm{CNC}$ can act in the crystallization kinetics. From the experimental data supplied by the DSC curves we can obtain the $X_{t} v s$. time curves, which were fitted using the Avrami equation (Figure 2b), in order to obtain the kinetic parameters ( $K$ and $n$ ), which are shown in Table 1.
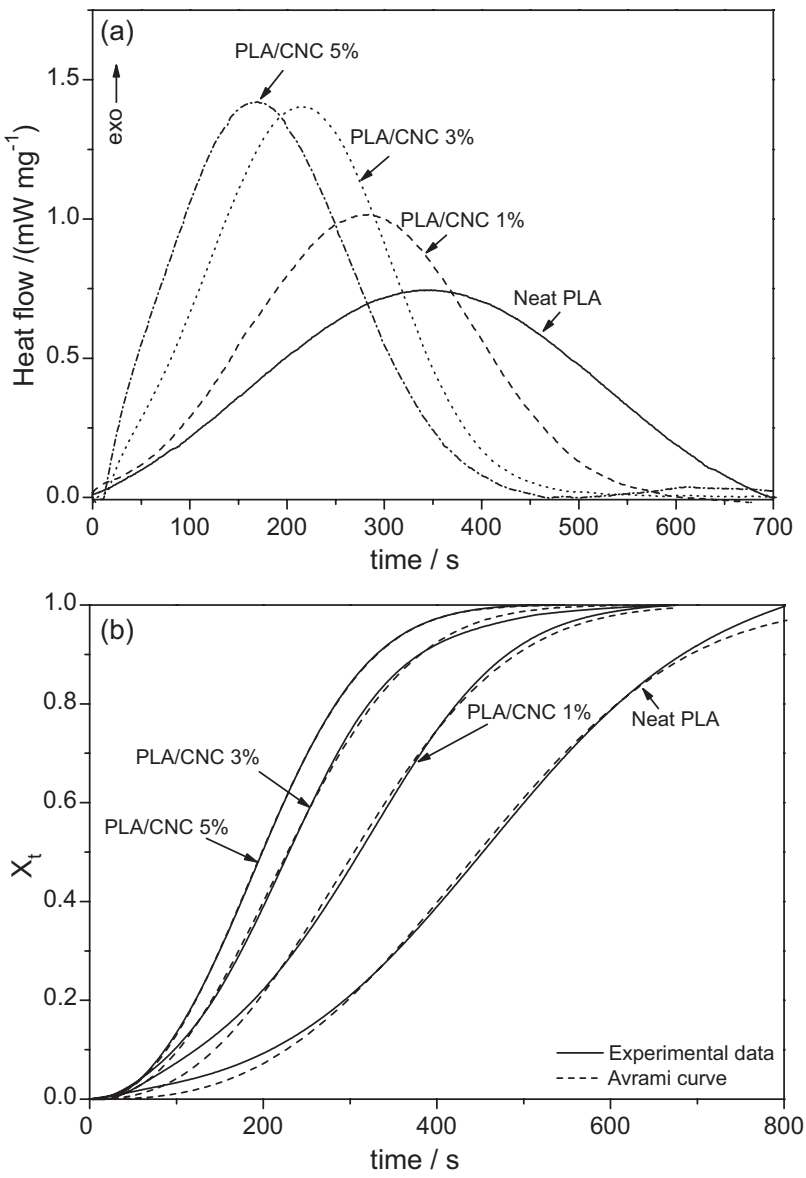

Figure 2. (a) Isotherms for the crystallization and (b) Avrami plots for the neat PLA and the PLA/CNC nanocomposites with different compositions. 
Table 1. Avrami parameters and degree of crystallinity percentage for isothermal crystallization kinetics of PLA and PLA/CNC nanocomposites

\begin{tabular}{lccc}
\hline Sample & $K \times 10^{3} / \mathrm{s}^{-1}$ & $n$ & $X_{c}^{\infty} / \%$ \\
\hline Neat PLA & 1.95 & 2.70 & 74 \\
PLA/CNC 1\% & 2.84 & 2.51 & 71 \\
PLA/CNC 3\% & 3.75 & 2.34 & 60 \\
PLA/CNC 5\% & 4.31 & 2.34 & 57 \\
\hline
\end{tabular}

$K$ : Avrami parameter; $n$ : Avrami exponent; $X_{c}^{\infty}$ : degree of crystallization; PLA: poly(lactic acid); CNC: cellulose nanocrystals.

The $K$ parameter has a physical meaning and expresses the rate of the crystallization process as a function of the nucleation and growth rate. A significant increase in the $K$ parameter was observed when the CNC content increased and the data indicate that only an increase in the $\mathrm{CNC}$ accelerates the crystallization process. Based on this result it is not possible to affirm whether the $\mathrm{CNC}$ are more effective as a nucleating agent or if they have an effect on the growth rate. The Avrami exponent $n$ is dependent on the nucleation mechanism or growth dimensions, ${ }^{30}$ and according to Wunderlich ${ }^{31}$ and Wenig et al., ${ }^{32}$ a decrease in the exponent $n$ by a value of one may be associated with a change from thermal to athermal nucleation. A decrease in the $n$ parameter may reflect a change in the nucleation mechanism to athermal heterogeneous nucleation. ${ }^{33}$ Intermediate $n$ values may be associated with a combination of mechanisms, since heterogeneous nucleation becomes more or less frequent with an increase in the heterogeneous nuclei, i.e., as the $\mathrm{CNC}$ content increases, up to saturation (above $3 \% \mathrm{~m} / \mathrm{m} \mathrm{CNC}$ ), after which changes in the exponent $n$ were not observed. To verify whether the presence of $\mathrm{CNC}$ accelerates the crystallization process only through a nucleation effect, optical microscopy with polarized light (Figure 3) was performed under the same isothermal crystallization kinetics conditions, in order to evaluate the growth of spherulite crystals in the different components of the composition.

The diameters of the spherulites were measured and plotted vs. time (Figure 4a). Based on the linear section of the curve, before impingement (Figure $4 \mathrm{~b}$ ) it is possible to determine the growth rate. No significant change was observed in the growth rate of the spherulites and the difference between the slopes for the PLA and PLA/CNC nanocomposites is within the standard deviation of the spherulite size, in agreement with results for poly(oxyethylene) (POE)/CNC reported by Samir et al. ${ }^{15}$ The Avrami $K$ parameter is dependent on the growth and nucleation rate. As observed through experimental data, the presence of $\mathrm{CNC}$ has no significant effect on the growth rate
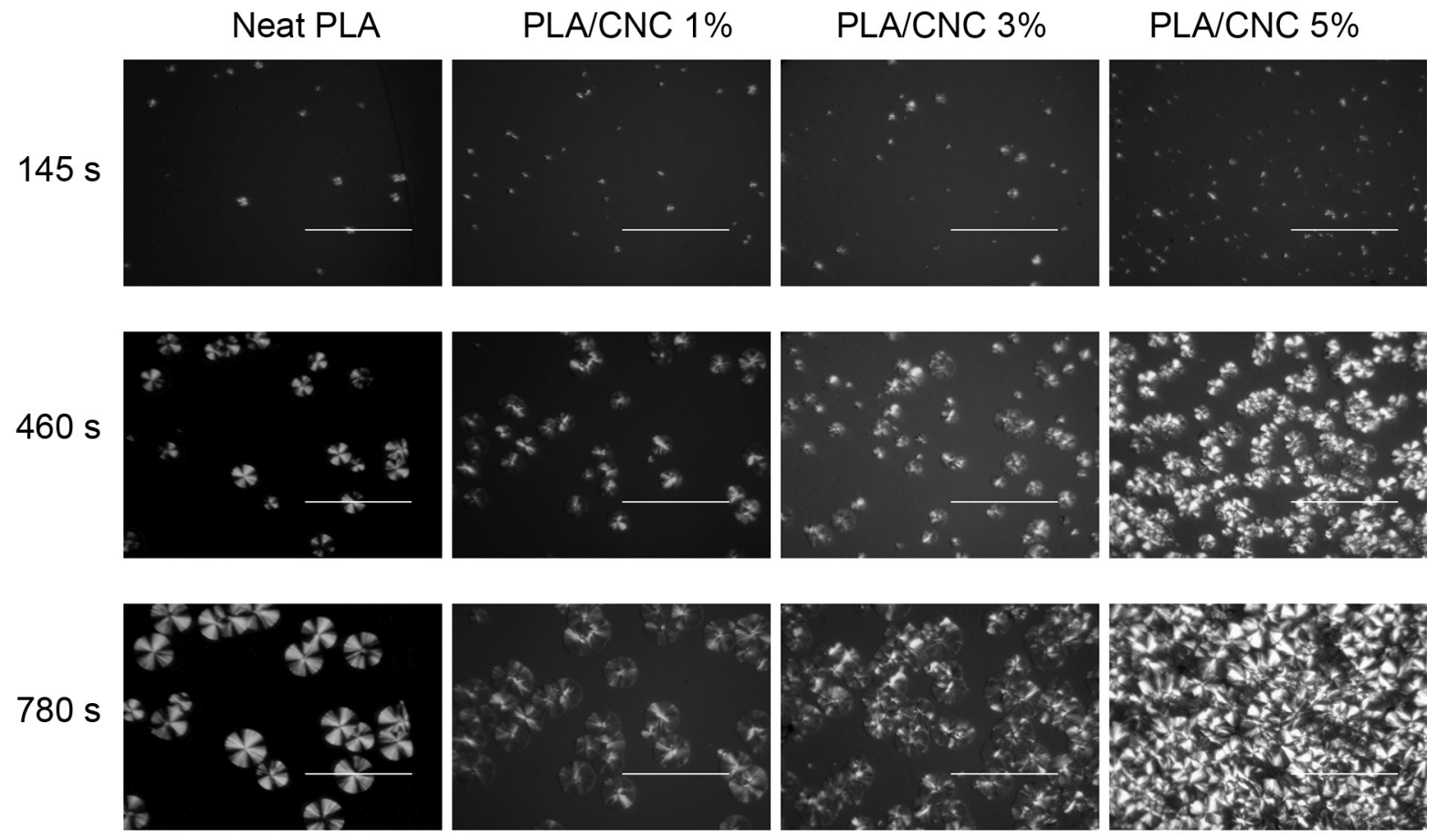

\section{$300 \mu \mathrm{m}$}

Figure 3. Optical micrographs obtained during isothermal crystallization of neat PLA and PLA/CNC nanocomposites at different time intervals (scale bar: $300 \mu \mathrm{m}$ ). 
and this observation is associated with an increase in the number of crystallization nuclei, as can be seen in Figure 3, suggesting that the increase in the rate of crystallization is only associated with the effect of the $\mathrm{CNC}$ on the nucleation of PLA crystals.
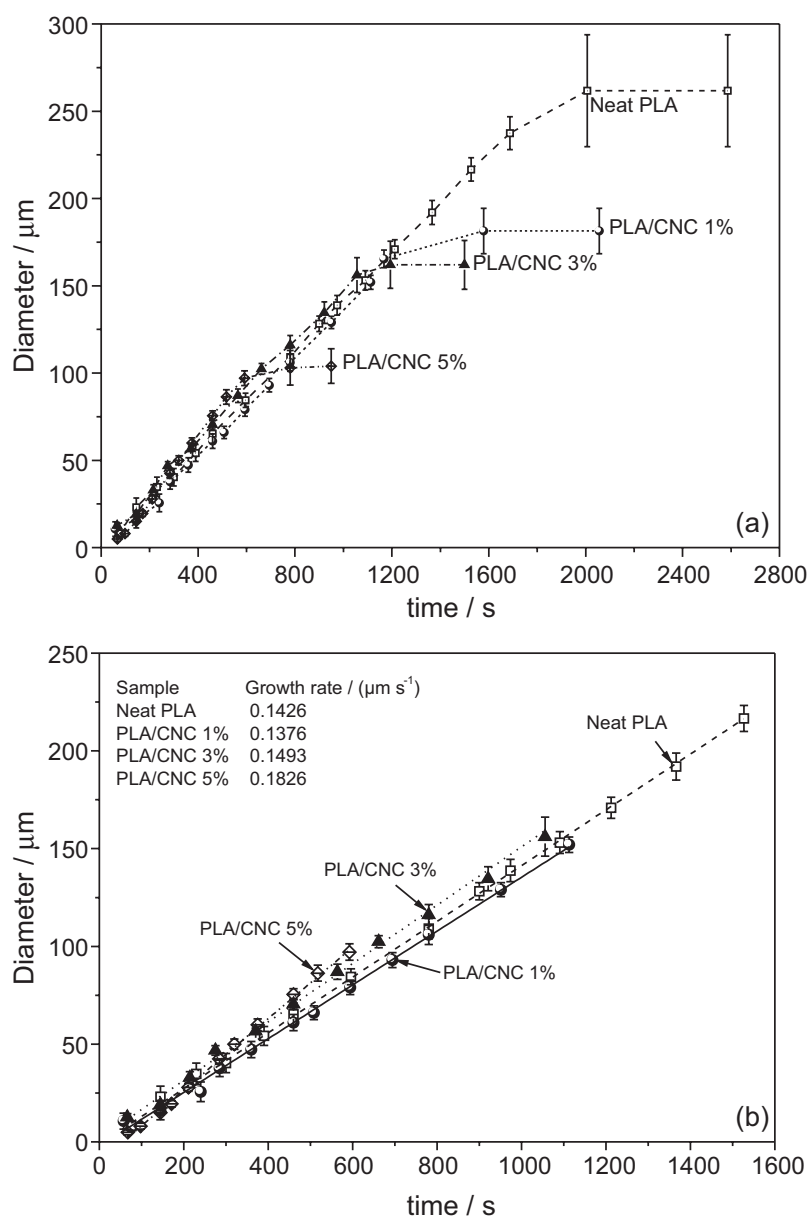

Figure 4. (a) Diameter of spherulites in neat PLA and PLA/CNC nanocomposites $v s$. time and (b) linear section of the curve before impingement.

The degrees of cyrstallinity obtained using equation 1 (reported in Table 1) show that there is a decrease in crystallinity with increasing $\mathrm{CNC}$ content. An increase in the content of CNC in the PLA matrix has a direct influence on the number of crystallization nuclei (Figure 3 ), which causes the impingement of spherulites to occur earlier and this affects the final size of the spherulite crystals. The number of spherulites per volume of polymer increases as the $\mathrm{CNC}$ content increases, which results in an increase in the volume of the spherulite border and, therefore, in a higher relative amount of the amorphous fraction, as shown in Table 1, where a reduction in the degree of crystallinity with increasing CNC content can be observed.

Figure 5 shows the XRD patterns for the PLA and films of PLA/CNC with 1, 3 and $5 \%$ of CNC, prepared isothermally at $130{ }^{\circ} \mathrm{C}$. It can be observed that the same set of $2 \theta$ angles of $12.5,14.8,16.8,19.1$ and $22.3^{\circ}$ appears for all samples, which are related to the families of crystalline planes: (103), (100), (110)/(200), (203) and (015), in agreement with results reported by other authors. ${ }^{34-36}$ This provides evidence of the presence of a single $\alpha$ phase with the same lattice parameters characteristic of the orthorhombic unit cell, for all CNC contents investigated in this study.

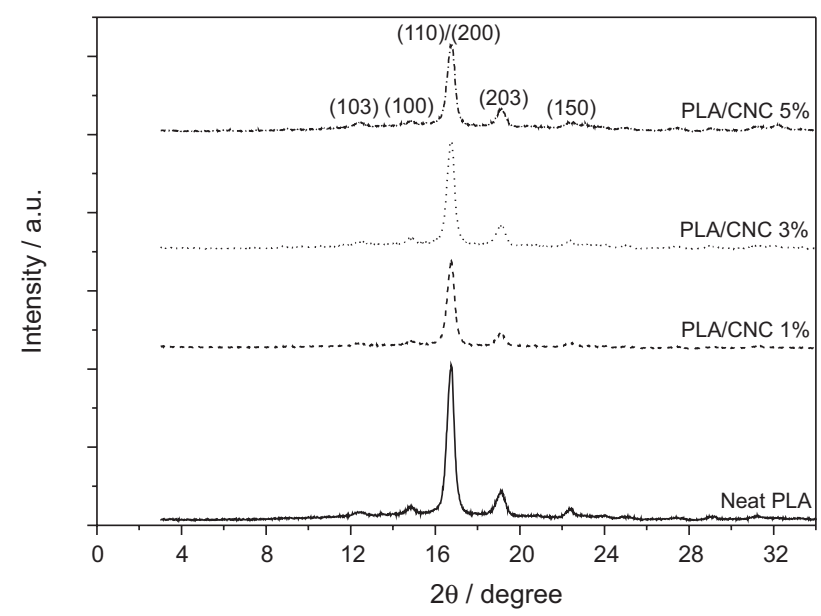

Figure 5. X-Ray diffraction patterns for samples of neat PLA and PLA/CNC nanocomposites with 1,3 and $5 \%(\mathrm{~m} / \mathrm{m}) \mathrm{CNC}$, after isothermal crystallization at $130^{\circ} \mathrm{C}$.

\section{Conclusions}

The CNC isolated in this study had the dimensions and morphological characteristics of nanometric structures. The CNC can act as PLA nucleating agents, affecting the density of nucleation, thus decreasing the final size of the spherulites as well as the rate of crystallization and degree of crystallinity of the PLA. However, no significant effect of the presence of CNC on the growth rate of the PLA spherulites was observed. In addition, the presence of CNC did not affect the lattice parameters of the PLA crystals at the CNC contents investigated in this study.

\section{Acknowledgments}

The authors are grateful for the financial support provided by CAPES and CNPq and also for the characterization by transmission electron microscopy performed by LCME-UFSC.

\section{References}

1. Reddy, M. M.; Vivekanandhan, S.; Misra, M.; Bhatia, S. K.; Mohanty, A. K.; Prog. Polym. Sci. 2013, 38, 1653. 
2. Refaa, Z.; Boutaous, M.; Rousset, F.; Fulchiron, R.; Zinet, M.; Xin, S.; Bourgin, P.; AIP Conf. Proc. 2014, 1593, 342.

3. Habibi, Y.; Lucia, L. A.; Rojas, O. J.; Chem. Rev. 2010, 110, 3479 .

4. Moon, R. J.; Martini, A.; Nairn, J.; Simonsen, J.; Younblood, J.; Chem. Soc. Rev. 2011, 40, 3941.

5. Auras, R.; Harte, B.; Selke, S.; Macromol. Biosci. 2004, 4, 835.

6. Hoogsteen, W.; Postema, A. R.; Pennings, A. J.; Brinke, G.; Macromolecules 1990, 23, 634.

7. Garlotta, D. A.; J. Polym. Environ. 2001, 9, 63.

8. Gupta, A. P.; Kumar, V.; Eur. Polym. J. 2007, 43, 4053.

9. Petersson, L.; Kvien, I.; Oksman, K.; Compos. Sci. Technol. 2007, 67, 2535.

10. Suryanegara, L.; Nakagaito, A. N.; Yano, H.; Compos. Sci. Technol. 2009, 69, 1187.

11. Sanchez-Garcia, M. D.; Lagaron, J. M.; Cellulose 2010, 17, 987.

12. Mathew, A. P.; Dufresne, A.; Biomacromolecules 2002, 3, 609.

13. Anglés, N. M.; Dufresne, A.; Macromolecules 2000, 33, 8433.

14. Samir, M. A. S. A.; Chazeau, L.; Alloin, F.; Cavaillé, J. Y.; Dufresne, A.; Sanchez, J. Y.; Electrochim. Acta 2005, 50, 3897.

15. Samir, M. A. S. A.; Chazeau, L.; Alloin, F.; Sanchez, J. Y.; Dufresne, A.; Polymer 2004, 45, 4149.

16. Yu, H. Y.; Qin, Z. Y.; Liu, Y. N.; Carbohydr. Polym. 2012, 89, 971.

17. Camarero-Espinosa, S.; Boday, D. J.; Weder, C.; Foster, E. J.; J. Appl. Polym. Sci. 2015, 132, 41607.

18. Sanchez-Garcia, M. D.; Lagaron, J. M.; Cellulose 2010, 17, 987.

19. Petersson, L.; Oksman, K.; Compos. Sci. Technol. 2006, 66, 2187.
20. Fukuzumi, H.; Saito, T.; Wata, T.; Kumamoto Y.; Isogai, A.; Biomacromolecules 2009, 10, 162.

21. Kamal, M. R.; Khoshkava, V.; Carbohydr. Polym. 2015, 123, 105.

22. Bondeson, D.; Oksman, K.; Compos. Interfaces 2007, 4, 617.

23. Kim, D. Y.; Nishiyama, Y.; Kuga, S.; Cellulose 2002, 9, 361.

24. Araki, J.; Wada, M.; Kuga, S.; Langmuir 2001, 17, 21.

25. Pracella, M.; Haque, M. U.; Puglia, D.; Polymer 2014, 55, 3720.

26. Oksman, K.; Mathew, A. P.; Bondeson, D.; Kvien, I.; Compos. Sci. Technol. 2006, 66, 2776.

27. Samir, M. A. S. A.; Alloin, F.; Sanchez, J. Y.; El Kissi, N.; Dufresne, A.; Macromolecules 2004, 37, 1386.

28. Teixeira, E. M.; Bondancia, T. J.; Teodoro, K. B. R.; Corrêa, A. C.; Marconcini, J. M.; Mattoso, L. H. C.; Ind. Crops Prod. 2011, 33, 63 .

29. Mandal, A.; Chakrabarty, D.; Carbohydr. Polym. 2011, 86, 1291.

30. Ozawa, T.; Polymer 1971, 12, 150.

31. Wunderlich, B.; Macromolecular Physics, vol. 2; Academic Press: New York, 1976.

32. Wenig, W. H.; Fiedel, W.; Scholl, A.; Colloid Polym. Sci. 1990, $268,528$.

33. Cebe, P.; Hsiao, B. H. In Polymeric Materials Encyclopedia, vol. 8; Salamone, J. C., ed.; CRC Press: Boca Raton, 1996, pp. 6185.

34. Pan, P.; Liang, Z.; Zhu, B.; Dong, T.; Inoue, Y.; Macromolecules 2009, 42, 3374.

35. Lai, W. C.; J. Phys. Chem. B 2011, 115, 11029.

36. Xu, T.; Zhang, A.; Zhao, Y.; Han, Z.; Xue, L.; Polym. Test. 2015, 45,101 .

Submitted: August 6, 2015

Published online: December 3, 2015 